\title{
Entre Vierge et Quimbois: Délire religieux dans La Vierge du Grand Retour
}

\author{
Françoise du Rivage \\ Augsburg College, Minneapolis
}

$\mathrm{Au}$ lendemain de la guerre, alors que la Martinique se débat dans de graves problèmes économiques et que les grèves se multiplient sur les plantations de canne à sucre, on annonce l'arrivée dans l'île d'une vierge rédemptrice suscitant des miracles extraordinaires. Ainsi se résume le roman de Raphael Confiant qui dénonce cette vierge car la religion est depuis toujours l'arme des grands blancs pour faire taire les noirs et les faire travailler. Mais, s'il y a bien démystification, ce n'est pas dans le but de rejeter toute religion car croire est le propre de l'âme antillaise, ce qui fait à la fois sa faiblesse et sa force. Aussi la Vierge, tout en mettant en évidence le mensonge misérable des colons est en même temps un hymne à la foi antillaise, une foi unique à ce peuple. Produit de son histoire, celle-ci se caractérise par un syncrétisme qui met sur le même plan croyance en Dieu et au Quimbois et une intensité d'émotion qui va jusqu'au délire.

Il ne fait pas de doute que Confiant s'attaque dans ce roman au rôle qu'a joué historiquement la religion catholique aux Antilles. Religion de l'Autre, du colonisateur, elle représente avant tout sa loi et sa culture. Car enfin de quelle couleur est ce Dieu qu'adore l'Antillais catholique? Dictionneur force Philomène à se poser la question:

-Tiens ! Ouvre donc tes missels, va à l'église ou alors calcule dans ta tête et tu verras qu'ils n'ont pu qu'être blancs et bien blancs. TOUS! Depuis Abraham, depuis Moïse, depuis Saint Michel, depuis Joseph jusqu'à Saint Paul, ils ont tous des peaux roses de bébé." "Hon! finit-il par lâcher au bout d'un moment. C'est à savoir si Dieu le père lui-même n'est pas un aryen. (79)

La religion catholique permet d'imposer aux noirs la morale et la culture des blancs. Ainsi à une époque où "la marchandise d'En-France avait plus de prestige que la marchandise pays"(47), les Antillais sont-ils tout particulièrement réceptifs à cette vierge made-in-France. 
Or, la venue la Vierge du Grand Retour est avant tout une "manigance de la caste béké"(66) qui a pour but de rendre les noirs plus dociles. Elle n'est finalement qu'un substitut du fouet. Nous sommes en effet en 1948, la Martinique est maintenant un département et non plus une colonie et ce que les grands planteurs n'ont plus l'espoir d'obtenir par la force: soumission et travail dans les champs de canne, ils espèrent encore l'obtenir par la religion. Ainsi les instigateurs de la venue de la Vierge du Grand Retour sont-ils des êtres immoraux, l'évêque le premier. Chacun a son propre agenda, politique pour Bertrand Mauville, économique pour les békés. La venue de cette Vierge est une vaste tromperie à l'issue de laquelle le nègre devrait reprendre docilement le chemin des champs de canne, les poches allégées de tous les dons faits à la Madone. Le dépouillement est orchestré sur une grande échelle et rappelle l'organisation d'un hold-up avec camion à dix roues, argent entreposé en secret, etc. Un hold-up dont le "parrain" est l'évêque et dont les sbires sont les grands planteurs.

Cependant, la critique de la religion se fait surtout au niveau littéraire avec la remise en cause du texte écrit comme faisant loi. En effet, l'Ancien et le Nouveau Testament, en dépit de leur origine orale et des voix multiples qui les composent, sont lus comme s'ils avaient un auteur unique: Dieu. Le texte de Confiant subvertit cette pratique en utilisant les mêmes subdivisions que le texte sacré: Genèse, Exode, etc... mais en intercalant les passages en italique qui mettent en scène Yahvé et un texte en caractère plain qui présente des voix multiples et ainsi multiplie les points de vue, alternant la première et la troisième personne, mais aussi l'époque: ici 1948, là 1942, l'époque de l'Amiral Robert. Enfin, le ton des passages de Yahvé change de l'un à l'autre. Par exemple si dans la Genèse les renversements font sourire, dans l'Exode un style héroïque décrit l'asservissement des noirs des plantations et leur exode vers l'En- ville (Fort-de- France) et le morne Pichevin. Dans Lettres Créoles, décrivant la même alternance chez l'auteur haitien Frankétienne, Confiant et Chamoiseau notaient que "les textes en italique relèvent de l'ordre du récitatif tandis que les textes à caractère droit relèvent du narratif' (179). L'alternance d'un prétendu texte sacré et du texte narratif se trouve concentrée dans L'homélie prophétique de Philomène où alterne du latin d'église et un bref historique de la condition des nègres montrant comment la religion permet aux blancs et même aux noirs de justifier tous les malheurs de la race noire, de l'esclavage aux catastrophes naturelles (88).

Confiant lui-même remarque que la version qu'il nous présente est l'une parmi d'autres. Après avoir raconté la rencontre de Dictionneur et de Josépha Victoire, l'institutrice il remarque: "ceci n'est que l'une des multiples versions de leur rencontre" (51) Du duel de Philomène avec Dame Victoire et du miracle qui s'en suit il y aura aussi maintes versions que le narrateur nous communique car" (...) ce formidable bouche à oreille créole qu'est radio-bois-patate tourneboula l'affaire, comme à son habitude, dans trente-douze mille directions et amplifia son caractère miraculeux. " (105) Comment ne pas remettre en question le texte écrit lorsqu'on peut apercevoir son origine orale!

Qui dit religion dit prophéties. Or, le texte de Confiant multiplie les prophètes douteux. Dictionneur le premier remarque que nous sommes en 1948, donc que cela fait exactement un 
siècle que l'esclavage a été aboli et qu'une sacrée belle surprise" se prépare (57). Et fils-du-Diable, lorsqu'il a trop bu, n'a-t-il pas l'habitude de dire que si les dirigeants français n'abolissent pas le travail'il y aura un gigantesque tremblement de terre et la Montagne Pelée va se réveiller" (117). Celui qui dit s'appeler Cham, un échappé de l'hôpital psychiatrique de Colson qui a certainement emprunté son nom à la Bible bien que sa religion prétende plutôt à une origine africaine, prophétise la fin du monde au nom de sa religion..." (204) Les deux doigts levés de la Vierge du Grand Retour sont aussi prophétiques de la fin du monde toute proche: "Deux ans! Deux ans qu'il reste au genre humain à vivre en ce bas monde" (300) Toute religion demande à ses croyants de renoncer à tous les autres dieux, toutes unifient derrière une prophétie. Le texte de Confiant, lui, fait naître le doute par la multiplicité et la contradiction.

La remise en question de la religion se fait aussi par l'ironie. Confiant utilise, par exemple, le latin d'église dans un contexte profane. Florentin Deshauteur proclamait "urbi et orbi" "Moi j'ai été un dissident" (18), Cicéron Nestorin qui a abandonné sa médecine est appelé "l'ex-disciple d'Hyppocrate" (210) et la notion de miracle est remise en question avec la tâche blanche sur la peau de Fils-du-Diable-en personne ou la chinoise aux yeux bleus.

Enfin la critique se trouve dans l'aspect carnavalesque de la procession que Dictionneur et d'autres qualifient de "maquaquerie." Le Carnaval commence avec la création d'un comité d'organisation dont le comité de la Vierge du Grand retour est bien sûr une parodie puisqu'il mélange bien noirs et blancs mais dans des proportions très inégales et avec une majorité de blancs immoraux aux intentions sordides.

L'arrivée de la Vierge dans l'île se fait dans une atmosphère de carnaval avec fleurs, guirlandes électriques, arcs de triomphe et croix éclairées, feux d'artifice et foule immense. La Vierge elle même se trouve posée sur un char. Dans cette atmosphère carnavalesque la piété tourne à l'hystérie collective "Le détachement des policiers (...) a le plus grand mal à contenir la foule en délire. Déjà des négresses hystériques s'arrachent les cheveux et tombent du haut - mal, clamant leur foi (...)" (152).

Cependant le carnaval est plus qu'une exagération, il est symbolique du brassage des races et des cultures et des renversements sociaux. Celui-ci est en effet une fête folklorique relativement récente à l'origine blanche mais dont les noirs se sont emparés et où ils ont introduit massivement des éléments de leur culture et de leur religion (Bastide 194). C'est donc le roman tout entier qui acquiert une forme carnavalesque où tout est renversé et où la religion est un extérieur qui cache les agendas de chacun et les croyances les plus diverses.

Les personnages du roman seront donc pour la plupart doubles selon la tradition carnavalesque. Ainsi, Dame Josépha Victoire," l'institutrice, le samedi venu, oubliait en effet son maintient de bourgeoise, son quant-à-soi de chrétienne assidue aux offices et s'encanaillait au 'Select-Tango' déguisée en courtisane, persuadée à tort , que nul ne la reconnaissait derrière son maquillage provoquant, sa robe rouge sang et son mouchoir de tête artistement noué". (51) $\mathrm{Au}$ petit matin elle troque cet accoutrement contre 'une robe empesée, un fichu de 
communiante" et le Select Tango pour la cathédrale de Fort -de France. Or, la double vie de cette dame n'et pas seulement hypocrisie mais contradiction fondamentale qui trouve son expression lorsqu'elle fait l'amour avec Dictionneur et qu'elle exprime son plaisir en créole et sa honte en français (185). L'évêque, "véritable sosie de Méphistophélès" est d'après Mélion de Saint- Aurel "à la recherche de chair fraîche" (63) Philomène, est putain la nuit et de jour vend des chapelets dans les rues de Fort-de France, habillée en religieuse. Michel, le fils de Saint- Aurel, a malgré la couleur de sa peau une telle affinité avec les noirs que son père se demande si Da Sissine, sa Nounou noire "ne l'avait pas tout bonnement métamorphosé en nègre" (125). Quant-à la Vierge du Grand Retour, les Martiniquais ne doivent recevoir que' "une reproduction de cette statue" (108) et son authenticité sera questionnée tout au long du roman. Ainsi Dictionneur est pris de soupçons lorsque la statue lui paraît beaucoup plus petite vue de près que lorsqu'il l'a aperçue pour la première fois. Les apparences sont donc bien trompeuses.

Richard D.E. Burton remarque que le carnaval dans l'oeuvre de Confiant est plus que "le mariage sublime et burlesque des contraires," il ne renverse pas car les catégories raciales et sociales sont déjà passablement brouillées dans l'En-ville, il intensifie plutôt une vie déjà carnavalesque elle-même (Burton 208-89). Cette exagération des contraires est pour Confiant un moyen supplémentaire de forcer son lecteur à remettre le texte en question.

Cependant, la Vierge ne se contente pas de remettre la religion en question. Le roman nous montre également comment les antillais vivent leur religion à travers les comportement, les paroles et les pensées des personnages. Or, dans ce pays où se mêlent des races et cultures multiples, la religion elle-même ne peut être qu'un syncrétisme. Aussi trouverons-nous dans ce roman des éléments religieux et des croyances appartenant à de multiples cultures: religions africaines et hindous, Vaudou, Quimbois.

Il y a par exemple dans le roman des éléments inspirés des religions africaines comme par exemple la croyance qui considère que la femme qui relève de couche est impure et la coutume africaine qui consiste à enterrer le cordon ombilical des nouveau-nés sous un arbre. ${ }^{1}$ On trouve également le culte des ancêtres qui se manifeste dans le culte des morts. Suivre la procession de la Vierge à travers la campagne est assez effrayant pour la population de l'île car à la nuit tombée "n'importe quel zombi égaré pouvait barrer la procession" (200) et lorsque "Le Père Ploquet planta l'une des croix sous un gigantesque fromager, (il) provoqua un frisson chez maints pèlerins" (243) C'est en effet sous les fromagers que les antillais croient que se rassemblent les zombis, les esprits errants des morts et ils évitent normalement ces endroits surtout à la tombée de la nuit. La transe, élément religieux africain que l'on trouve également dans le Vaudou se retrouve à la Martinique et dans le roman sous forme de crise d'hystérie chez les femmes. "Ces crises sont attribuées à l'action des quimboiseurs et expliquées par le pouvoir qu'ils ont de faire entrer des démons dans le corps de leur victime" (Bastide 155).

On trouve également des éléments de religion hindou, puisque les colons ont importé bon nombre de travailleurs hindous pour remplacer les noirs qui ne voulaient plus travailler sur les 
plantations après l'abolition de l'esclavage. Leurs traditions et croyances religieuses ont assez bien survécu car d'une part les hindous se marient entre eux comme on le voit dans le roman et d'autre part les colons ont favorisé la construction de leurs temples près des plantations dans l'espoir de les voir s'y implanter. Les hindous sont représentés dans le roman par le jeune Manoutchy. Or "ils adorent particulièrement, comme le remarque l'évêque, une sorte de déesse qu'ils nomment Mariémen à qui ils offrent des sacrifices d'animaux aux cours de cérémonies sataniques"(141). D'après Harold Turner, cependant, la plupart des hindous de Martinique sont catholiques aujourd'hui mais fréquentent, à coté de l'église catholique, leurs temples où ils pratiquent une religion syncrétique faite d'éléments hindous et catholiques. ${ }^{2}$

Les éléments empruntés du Vaudou sont eux aussi très nombreux. Le personnage de Philomène, sainte et prostituée fait bien sûr penser à la déesse Vaudou Erzulie. Cette "mamzelle féerique, toujours drapée dans une robe fourreau couleur de firmament" (14), n'affirme-t-elle pas elle-même :"Je suis Notre Dame des Sept Douleurs!"(104) Or Erzulie, déesse de l'amour est la patronne des prostituées. Elle est aussi assimilée à Notre-Dame des Sept Douleurs ou à la Vierge Marie. Gilberte Dorival remarque que "Haïti, où le vaudou s'est largement développé, faisant de la magie une institution nationale, a également beaucoup influencé la Martinique et la Guadeloupe. D'ailleurs, les Hougan, sorciers haïtiens, bôcos y jouissent d'une haute considération de nos jours" (Dorival, Yo Garéi, 104).

D'après Confiant c'est également dans le Vaudou qu'il faut voir l'origine du chiffre deux qui joue un rôle important dans le roman. La vierge du Grand retour en effet "Majeur et index scellés l'un à l'autre, (elle) indique le chiffre deux" (150). Ce chiffre deux se retrouve dans les jumeaux que la nièce de Philomène, Adelise, a porté dans son ventre miraculeusement pendant onze mois. On trouvera d'autre part deux jumeaux cités dans la lettre de l'évêque au cardinal qui montrent que les antillais "attribuent à ces jumeaux le don de porter chance à toute personne qui leur touche le crâne"(139). De ceci il faut rapprocher également l'importance des couples de personnages qui s'affrontent dans des duels: Rigobert / Bec en or, Dictionneur/ Bec-en-Or, Josépha/ Philomène. En effet, dans Lettres créoles, Confiant et Chamoiseau remarquent à propos des personnages de Frankétienne qui fonctionnent par couple "Cela est à mettre en rapport avec la tradition Vaudou des 'Marassa' ou jumeaux, terme Africain, probablement Fon, qui est conservé dans la liturgie de cette religion. La problématique de la gémélité est d'ailleurs une des données majeures permettant le décryptage d'une sémiotique proprement haïtienne. Alfred Métraux note que 'les jumeaux vivants ou morts sont investis d'un pouvoir surnaturel qui fait d'eux des êtres d'exception" (177). ${ }^{3}$

Le dernier type de syncrétisme et sans doute le plus important à la fois dans les Antilles et dans le roman est le Quimbois. "Ce mot quimbois, connu depuis le dix-huitième siècle signifiait à l'origine sortilèges, maléfices... mixtures subtiles devant apporter le succès, la guérison ou la mort d'un ennemi' Il désigne également (...) toutes les manifestations d'ordre surnaturel (...) Le quimboiseur est celui qui pratique le quimbois mais le séancier (voyant-guérisseur) peut devenir quimboiseur. ${ }^{64}$ Or le quimbois, s'il est empreint de culture africaine a été influencé 
également par le Grand et Petit Albert ${ }^{5}$ et surtout par le christianisme. A la différence du Vaudou ou des cultes hindous le quimbois n'est pas à proprement parler une religion organisée. En outre, il occupe une place à part car il obéit à la loi de la magie qui est l'efficacité" (Bastide 165). Gilberte Dorival montre dans son ouvrage Yo Garéï, l'importance que joue l'imagerie religieuse catholique pour les séanciers et les quimboiseurs: les hosties, l'eau bénite, les chapelets dûment magnétisés, la Vierge, les saints martyrs, les croix, les prières catholiques sont utilisés abondamment par les séanciers et les quimboiseurs. "Tous les éléments de la religion chrétienne sont passés au crible des croyances africaines, C'est leur compréhension et la façon de les interpréter et de les associer qui changent" (Dorival 105). La religion catholique et la voyance ne sont donc ni séparés ni antithétiques."Parmi les croyances de type magique, il faut mentionner la numérologie, dont on trouve d'autre part des traces dans le Nouveau Testament, dans l'Apocalypse notamment. Outre la Vierge dont les deux doigts prophétisent la fin du monde, on peut noter qu'au Morne Pichevin les habitants croient que la septième marche porte malheur et "la trente-troisième est celle de la mort subite dans la fleur de l'âge" (192).

La magie a d'autre part la propriété d'utiliser le verbe non en tant que signifiant, mais pour sa force elle même. Pour les personnages du roman, les mots peuvent avoir une puissance magique. Ainsi dans la rixe qui l'oppose à Dictionneur, Bec-en-Or, devant un mot qu'il ignore se trouve désarmé car il ne sait si ce mot est une insulte ou "quelque conjuration maléfique" (53).

L'histoire d'Adelise et de sa grossesse, centrale dans le roman est affaire de quimbois. Lorsque Philomène réalise que la grossesse de sa nièce se complique et que la vie de cette dernière est en danger, Rigobert émet l'hypothèse que"un nègre de mauvaise vie lui a tout bonnement amarré l'enfant dans le ventre" (234) ce qui la fait trembler car ce qu'il veut dire c'est qu'on lui a envoyé un quimbois. Ce n'est donc pas au docteur qu'elle va s'adresser, mais au prêtre, magie et religion catholique étant liées dans l'esprit antillais. Elle compte sur l'achat de trois messes d'action de grâce pour rompre le quimbois. Lorsque le prêtre les lui refuse, elle réalise qu'il faut que sa nièce dévoile l'identité du père de l'enfant car il est sans doute à l'origine du quimbois. "Une fois dévoilé l'identité du père de l'enfant, Philomène lui ferait rendre gorge, l'obligerait à défaire le 'mal' qu'il avait envoyé sur sa nièce" (34).

Pour les pèlerins qui suivent la madone, la religion catholique est une forme de magie comme le montre l'épisode suivant: Le quimboiseur Chrisopompe a la réputation d'être capable de faire disparaître d'un seul mot les gens qui s'opposent à lui, en particulier les femmes qui se refusent à lui. Lorsqu'il annonce qu'il fera disparaître les pèlerins et la Madone aux Mornes-des-Esses, beaucoup le croient et sont terrifiés. Lorsqu'il est mis en déroute par le père Le Gloarnec, la foule des campagnards qui observait la scène, médusée s'écria"le Blanc-France est le plus fort, oui!"(257) La foi des pèlerins se trouve renforcée car la magie des Blancs l'a emporté pour eux sur celle des noirs, sur les croyances africaines héritées des nègres Congo. 
C'est parce que religion et magie sont si intimement liées aux Antilles que les personnages du roman attendent de la religion et donc de la Vierge du Grand Retour l'efficacité. Ainsi, le miracle s'il est exceptionnel dans la religion catholique va de soi dans les religions noires. Roger Bastide remarque que "le nègre n'a pas une religion de crédit (accumuler les bonnes actions sur la terre pour être récompensé dans le ciel) Il veut obtenir satisfaction hic et nunc ; ce que le père divin annonce c'est le royaume de Dieu sur la terre: "Chaque jour nous avons ici la puissance du ciel" (Bastide 170). Dame Victoire qui connait bien ses compatriotes s'inquiète qu'ils ne demandent trop de miracles à la Vierge mais avec l'exagération carnavalesque de Confiant, elle a tort de s'inquiéter car sa congénère Philomène lui fait aussitôt remarquer: "Je vous demande pardon . J'ai assisté en personne à quatre miracles hier soir sur la Savane" (186).

C'est d'autre part parce que leur religion est efficace que pour les personnages du roman religion et politique sont intimement liées. Le moment historique où prend place le roman, après le départ de l'Amiral Robert, au début de la départementalisation explique que le peuple antillais soit en attente d'un ordre nouveau. Ainsi, comme Jésus était un nouvel Adam et représentait pour les juifs la possibilité d'un nouveau début, les antillais attendent, au lendemain de la guerre, un changement majeur qui renverserait l'ordre établi ne laissant aucun espoir aux noirs. Les antillais, comme bien d'autre peuples oppressés en Amérique, voient une similitude entre la colonisation des blancs et l'oppression de Pharaon sur le peuple juif dans la Bible, d'où l'attente d'un messie qui viendrait les libérer. Qui plus est, l'histoire des peuples noirs a vu, avec le Vaudou, le rôle que pouvait jouer la religion dans la libération d'un peuple. Or il s'agit justement d'une religion syncrétique d'origine africaine et qui a montré une adaptation à la réalité américaine. ${ }^{6}$

Outre l'efficacité, il faut remarquer que la religion des personnages du roman est plus affective que rationnelle ou morale. Ceux-ci demandent des signes extérieurs: visions, rêves, transes et cultivent la part sentimentale de l'âme (Bastide , 208). D'après Roger Bastide, si la religion du Noir américain est affective, ce n'est pas à cause d'une survivance africaine, mais parce que l'esclave exploité avait besoin de trouver une compensation voire un défoulement dans le christianisme (Bastide 208).

Parce qu'elle relève si fortement de l'émotion, la "foi" des personnage va souvent jusqu'à la folie. Rigobert au moment du départ de la Vierge parle de "pèlerinage démentiel" (304), décrivant un pèlerinage ou le comportement des personnage peut être qualifié de délirant. Philomène apparaît hagarde. "c'est la fixité de son regard qui effraie surtout Rigobert" (306). Elle est comme absente, vidée d'elle-même" (308) Cependant, de nombreux autres personnages sont eux aussi en proie à des moments de délire au cours de la procession. Dans son ouvrage Yo Garéi, Gilberte Dorival montre à la fois la fréquence des délires religieux aux Antilles et les relations entre quimbois et maladie mentale. En particulier il n'est pas rare que les malades mentaux croient entendre des voix, à l'exemple de Philomène ou de Dictionneur. Pour Dorival, le séancier joue le rôle de thérapeute et est en général consulté par le malade 
mental et sa famille avant les médecins occidentaux. Cependant celle-ci le fait en secret car "La fréquentation des séanciers est mal vécue aux Antilles, c'est pourquoi on les visite dans la plus grande discrétion. Les consultants ne savent plus s'ils font le bien ou le mal et se culpabilisent" (Dorival 238). D'après Dorival il n'y a pas aux Antilles de rites d'initiation semblables à ceux des religions africaines "Par contre, l'initiation est présente sur le modèle du chamanisme" (Dorival 139). Le séancier en proie à un délire religieux, est lui-même malade mais capable de donner un sens à ses symptômes en l'inscrivant dans une filiation renvoyant aux origines. Ainsi est-il capable d'aider les autres à interpréter leurs rêves ou à renvoyer un quimbois. Conflits et sentiments refoulés s'expriment à travers rêves, angoisses, délire. Ces symptômes peuvent disparaître si la personne qui les éprouve croit qu'elle est victime d'un quimbois de la part de quelqu'un et lui envoie un contre-quimbois.

Les conflits qui apparaissent dans le roman sont souvent personnels. C'est le cas de Philomène par exemple. Celle-ci est fragilisée par la mort de l'homme qu'elle aime. Mais elle se trouve particulièrement déstabilisée par la grossesse de sa nièce à cause de sa propre stérilité. Bien qu'elle ne l'exprime jamais ouvertement, elle ne peut s'empêcher d'éprouver envie ou jalousie à son égard. Lorsque la grossesse se complique elle voit dans les complications la manifestation d'un quimbois. Ce quimbois, qui représente le conflit qui l'habite la plonge dans un délire religieux car, faute de savoir qui en est l'auteur, elle ne peut le renvoyer. Mais, chez Confiant, comme chez Chamoiseau, le délire exprime non seulement les conflits personnels des personnages mais surtout le malaise de la société. Ainsi Rigobert et Fils-du-Diable-en-personne, expriment-t-ils dans leur délire le voeu de devenir blanc ou d'avoir les yeux bleus, prêt à renoncer à leur identité pour ressembler à l'Autre qu'ils croient supérieur. Le délire de Dictionneur traduit le conflit entre son désir de ressembler au blanc qu'il croit supérieur —n'oublions pas qu'il refusera toujours de se séparer de son dictionnaire, symbole de la puissance des colons - et le désir de retrouver le passé africain de sa race. La difficulté de retrouver ce passé apparaît également dans le délire de Mathieu Salem. A ce moment clé de l' histoire des Antilles qu'est le début de la départementalisation, la société antillaise est partagée entre le désir de s'intégrer à la société française qu'elle croit supérieure et la peur d'être cannibalisée par la culture blanche. Au plus profond d'eux mêmes les personnages ne peuvent réconcilier les oppositions de leur culture: culture traditionnelle et religion catholique, français et créole, bien et mal. Se voyant avec les yeux du blanc ils assimilent leur culture au mal: si les noirs sont maudits c'est qu'ils sont mauvais, immoraux. Mais plus ils rejettent leur culture et plus elle les hante et devient symptomatique. Le refoulé ne peut plus s'exprimer que dans le délire ou le rêve. La dualité qui apparaît dans la symbolique religieuse (chiffre deux, jumeaux, etc.) ne peut être reeconciliée.

La Vierge du Grand Retour est symbolique de la religion aux Antilles. Son retour forcé lorsque la supercherie des blancs est découverte n'est peut être qu'un autre mauvais tour joué par la fortune. En effet la fin du roman, si elle semble satisfaisante pour tous (Les méchants sont punis et les personnages sont satisfait par la construction d'une église qui abritera leur 
vierge bien-aimée), ne saurait effacer l'image de la religion que présente Confiant dans le roman. Or celle-ci semble crystaliser tous les conflits de la culture antillaise. Il ne suffit pas de dénoncer la religion importée et imposée car celle-ci s'avère nécessaire. Cependant la Vierge du Grand Retour ne peut être la même que la Vierge de Boulogne, même s'il s'agit de la même statue. Même baptisée catholique, la religion aux Antilles se doit d'être re-définie commele démontre le roman de Confiant.

\section{Notes}

1 "Ainsi "Yahvé Dieu parla au Maître de grande Savane et lui dit: "Parle à ta servante Adelise dont tu as ravi la virginité et dis -lui: si elle est enceinte et accouche d'un garçon, elle sera impure pendant sept jours comme au temps de la souillure de ses règles Au huitième jour, tu feras venir une matrone qui ensevelira la corde du nombril du nouveau-né à l'ombre de l'arbre le plus imposant de a plantation. (...) Si elle enfante d'une fille, elle sera impure pendant deux semaines, comme pendant ses règles, et restera plus de soixante-six jours à purifier son sang." (68) On retrouve ici également la coutume africaine qui enterre les nombrils des nouveau-nés au pied d'un arbre et qui se trouvait déjà dans les paroles de Rigobert "Vous enterrerez les cordes du nombril de vos enfants nouveaux-nés à l'en-bas de ses racines!" (30)

2 "In Martinique the descendants of the Asian Indians imported as plantation labourers last centuy are now almost all Roman Catholics... Alongside this they have developed a number of cult centers with temples where individuals are able to secure several servicess when they nee personal help in matters of health, marriage, prosperity, etc.The services follow he sacrificial rituals to the gods in a ssouth Indian village but the deities have been identified with christian figures: the chief deity, Maldevidan or Vishnu is equated with Christ, and the goddess Mari-eman with the Virgin Mary, other deities correspond to various Christian saints." Harold Turner, New Religious Movements in the Caribbean, 56.

3 "The most common convergence of religious elements is in the association made between catholic Saints and african deities, so that in Haïti for exemple Legba, like Saint Peter is regarded as the keeper of the keys to the after life (...) The interpretation of Erzulie in Haiiti coincide with that of our Lady of Seven Sorrows." Elisabeth Thomas-Hope, The Pattern of Caribbean religion, 10.

${ }^{4}$ Le pouvoir surnaturel attribué aux jumeaux n'est pas limité aux cultures africaines ou au vaudou puisqu'on trouve cette croyance également en France au moyen âge et dans beaucoup d'autres cultures à travers le monde. 
${ }^{5}$ En effet, "la magie des blancs a été tenue par les noirs comme supérieure à la leur, puisque la leur n'arrivait pas à les délivrer de l'esclavage et que celle des blancs leur assurait une domination sans failles. C'est pourquoi sans renier leurs pratiques africaines, lorsqu'elles s'avéraierent efficaces, il y ajoutèrent les pratiques européennes, lorsque les premières ne réussissaient pas." Gilberte Dorival, Yo Garéï, 263.

${ }^{6}$ ouvrages de magie

7 "Le vodou joue son rôle de catalyseur de conscience, de générateur d'attitude socio-politiques cohérentes et bénéfiques pour le groupe national. Dans les arts, dans les lettres, l'influence vodou se fait de plus en plus sentir. Elle est une source d'inspiration merveilleuse, prodigieuse, originale pour peintres, poètes, sculpteurs, artisans, chorégraphes qui par ricochet travaillent au bénéfice d'un art spécifiquement national." Gerson Alexis, Vodou et quimbois, 55 .

\section{Ouvrages cités}

Alexis, Gerson. Vodou et Quimbois. Port-au-Prince (Haïti) : Éditions Fardin, Collection Lecture Anthropologique Haitienne, 1976.

Bastide, Roger. Les Amériques noires. Paris: L'Harmattan,1996.

Burton, Richard D.E. Le Roman marron. Paris: L'Harmattan, 1997.

Chamoiseau, Patrick et Confiant, Raphael. Lettres créoles. Paris: Hatier, 1991.

Confiant, Raphaël. La Vierge du Grand Retour. Paris: Grasset, 1996.

Dorival, Gilberte. Yo Garéi. Paris: L'Harmattan, 1996.

Gates, Brian, ed. Afro-Caribbean Religions. London: Ward Lock Educational, 1980. Thomas-Hope, Elisabeth."The Pattern of Caribbean Religions." Afro-Caribbean Religions. Brian Gates, ed. London: Ward Lock Educational, 1980.

Turner, Harold. "New Religious Mouvements in the Caribbean." Afro-caribbean Religions. Brian Gates, ed. London: Wardlock Educational, 1980. 